\title{
Analysis of service properties of cylindrically ground surfaces, using standard ISO 25178-2:2012 surface texture parameters
}

\author{
Natalija Bulaha \\ Riga Technical University, Institute of Mechanical engineering, \\ Address: Ezermalas street 6k, Riga, LV-1006, Latvia.
}

\begin{abstract}
At present surface roughness is one of the main indications of component quality. During the operation mainly the outer layer of the component is exposed to external action, and depending on the roughness structure conclusions can be drawn about the service properties of the surface. Because cylindrical grinding today is rather widely used and precise method of treatment of bodies of revolution in the field of mechanical engineering the given paper pays attention to the surfaces treated on cylindrical grinding machines, and more exactly to their roughness, determining the possible use of the given surfaces. For the analyses of the roughness parameters the ISO standard ISO 25178-2:2012 “Geometrical product specifications (GPS) - Surface texture: Areal - Part 2: Terms, definitions and surface texture parameters" was used, in which there were determined the texture parameters of the surface. Contrary to other standards, such as ISO 4287, ISO 13565 the new standard allowed to assess real surface properties, since in the given case the surface is being viewed in three-dimension space. During the research three cylindrically ground samples treated at different cutting conditions were chosen. By the help of modern measuring equipment Taylor Hobson Talysurf Intra 50 the topography of the given samples was taken in order to obtain and compare the roughness parameters and for the purpose of setting relation between the cutting regime and roughness parameters, and determining mathematical model of cylindrically ground samples. As a result of research it was stated that the given type of surfaces is characterized by irregular roughness, that is location of micro-peaks regarding their height and shape are irregular and also the correlation function of cylindrically ground surfaces is rapidly diminishing, but the density distribution of ordinates is close to normal. Based on the above feature of surface roughness such service properties as wear resistance, retention of lubricants and coatings (adhesion) and provision of required contact area were determined.
\end{abstract}

Keywords: Surface roughness, topography, cylindrical grinding, exploitational characteristics.

\section{INTRODUCTION}

Nowadays the volume of industrial production dramatically increases, due to the modernization of machine tools and equipment and thus enhance of their operational efficiency.

As it is known quality of manufactured details depends on technological factors, which to a greater or lesser extent affect the machining accuracy, and is characterized by a tolerance in size, shape, location and surface roughness. Particular interest is caused by the surface roughness, which helps to determine the service properties of machine components.

The present paper deals with the topography and parameters of surfaces treated on cylindrical grinding machines, and striking representatives are cylindrical and conic surfaces of components of type "shaft" and also internal surfaces. The given type of treatments is rather modern and sufficiently precise and therefore the determination of features of roughness of cylindrically grinded surfaces will help improve the service properties of machine components.

Exploitational properties of surfaces include mainly wear resistance, retention of lubricants and coatings and also provision of contact area, which is particularly important when choosing fit and ensuring hermeticity. All the above properties can be determined theoretically and practically using roughness parameters measured on special "profilograph - profilometer" type equipment.

The given paper for the surface analyses uses relatively new Standard ISO 25178-2:2012 [1], comprising 6 groups of texture parameters of surface: height, functional, spatial, hybrid, functions/related and miscellaneous parameters. In the given standard, 
as compared with others the roughness parameters allow to assess the actual properties of the surface, since their determination is carried out in three dimensional medium.

The following paragraphs will consider an experiment on the determination of roughness parameters of three cylindrically grinded surfaces and give analyses of their exploitational characteristics.

\section{CYLINDRICALLY GROUND SAMPLES AND MEASUREMENT METHODS}

For topography analyses of cylindrically grinded surfaces there were chosen surfaces No.1, 5, 8 (Fig.1) of Rugotest 105 sample (company L.C.A.-C.E.A.). Sample's material - corrosion resistant nickel treated on a cylindrical grinding machine at different feed rate; the sample being made according to ISO 2632 standard.

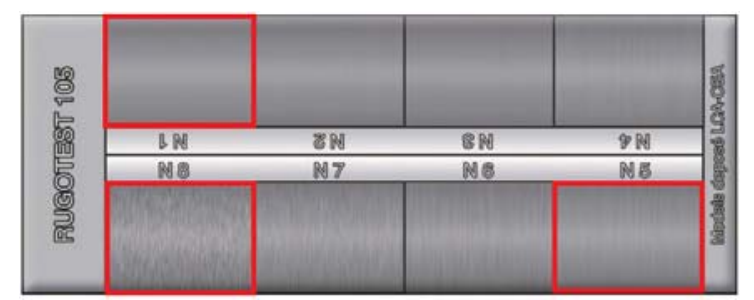

Fig.1. Cylindrically grinded sample Rugotest 105

The roughness of surfaces of the given sample was measured using modern profilograph - profilometer Taylor Hobson Talysurf Intra 50 (Fig.2), equipped with a stylus with a needle, drive, a table, a unit of electronic system to collect information and a computer necessary for data processing. The resolving power of the given roughness indicator is $16 \mathrm{~nm}$ for the $1 \mathrm{~mm}$ range. The roughness measurement principle is based on the movement of the probe-needle along the examined surface, whereas the needle prints down the profile of the roughness [2].

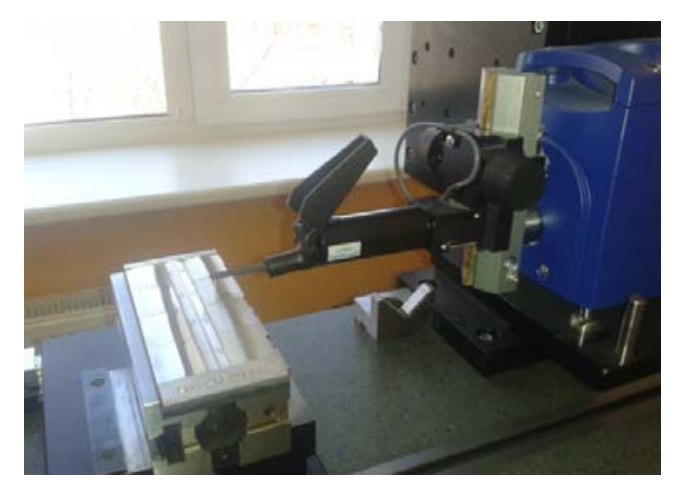

Fig.2. Measurement device Taylor Hobson Talysurf Intra 50

Because the profilograph - profilometer is equipped with 3D measurement system, which ensures movement of the table with the sample and measuring needle along three axes, to get a surface topography is a very simple task for this device.

For the determination of $3 \mathrm{D}$ roughness of the surface special software: TalyMap expert (3.2.0.Version) was used for the input of needed parameters and $\mu$ ultra - for the processing of surface topography.

\section{EXPERIMENT ON ROUGHNESS MEASUREMENT}

Prior to carrying out experiment it was necessary to clean the surface from oil and dirt using alcoholic solution. Then the sample was placed and fixed on the measurement table and the measurement needle was attached to it to ensure an initial contact. The pressing force of the needle was set according to the software data. Further in the software TalyMap expert the needed measurement conditions were chosen, which are shown in Table 1.

Table 1

Set conditions of measurement of roughness parameters in TalyMap

\begin{tabular}{|l|l|}
\multicolumn{2}{c|}{ expert software } \\
\hline Parameter & Value \\
\hline Number of points (Y) & 150 \\
\hline Data length (Y) & $2 \mathrm{~mm}$ \\
\hline Number of points (X) & 150 \\
\hline Data length (X) & $2 \mathrm{~mm}$ \\
\hline Measurement Speed & $0,5 \mathrm{~mm} / \mathrm{s}$ \\
\hline
\end{tabular}

When experiment was finished, initial topographies of all three surfaces were obtained. The term "initial" means that given topographies do not provide a possibility to determine actual values of roughness parameters. For this purpose it is necessary to process the obtained data [3], [4], separating from the initial topography the form and waviness, which are the result of wrong cutting regimes, vibrations during the operations and other factors. Additionally before filtration it is necessary to carry out leveling of the surface, because when sample is fixed on the table it is impossible to ensure a full contact area, and thus the measuring needle will not be situated towards the sample's surface at the right angle (Fig.3).

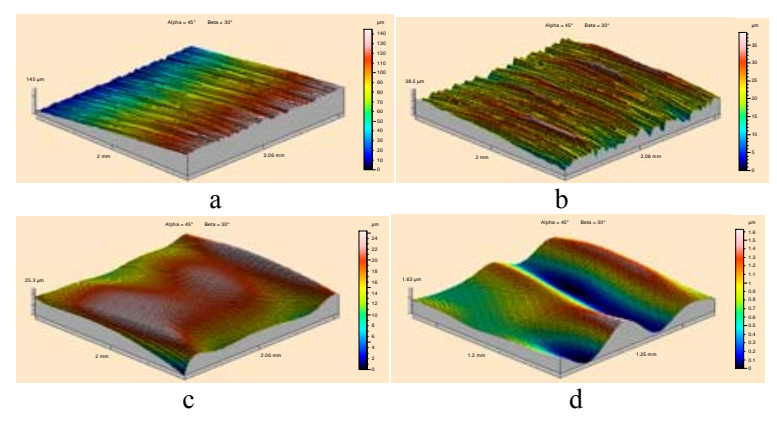

Fig.3. Surface topography filtration a - initial topography, b - topography after leveling, c - separated form of the surface, $d$ - separated waviness of the surface 
After the surface filtration the actual roughness was obtained, what is shown in Fig.4. The surface roughness after cylindrical grinding has parallel situated scratches caused by abrasive grains. Fig. 4 shows that roughness has a definite orientation, which demonstrates non-isotropy of surface, which influences the surface strength.

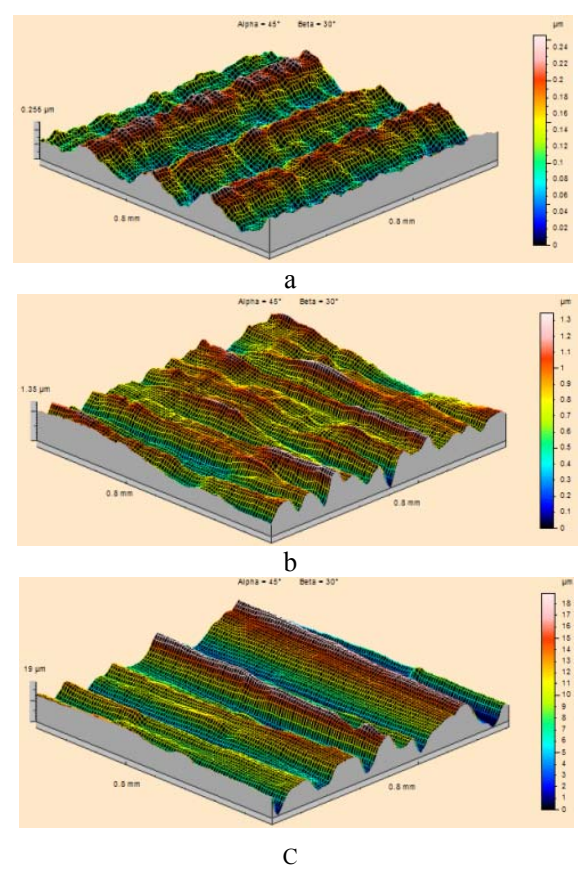

Fig.4. Roughness of surfaces No.1 (a), No.5 (b), No.8 (c)

Additionally there were determined the correlation function of roughness of cylindrically grinded samples, which indicates a connection between the process points [5], and also the density distribution of ordinates of the surface. The correlation functions of all three surfaces are diminishing with damped oscillations and the distribution density is close to normal and corresponds to Gaussian distribution law (Fig.5).

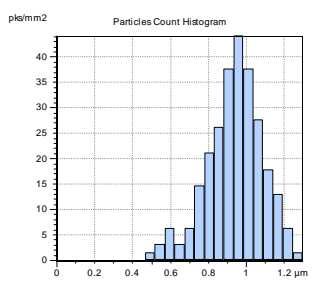

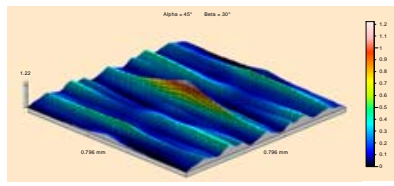

b
Fig.5. Correlation function of roughness (a) and density distribution of ordinates (b)

Further on in this paper there were determined roughness parameters of surfaces under research. For this purpose in $\mu$ ultra software it was necessary to choose the function "parameters", isolating previously the filtered topography. The obtained values differ from each other, what can be explained by varying of cutting conditions. Table 2 presents roughness parameters needed for the analyses.

Further in the paper the obtained roughness parameters will be examined more in details in order to determine the service properties of surfaces and relation between roughness parameters and cutting condition.

Table 2

Roughness parameters of surfaces №1, №5, №8

\begin{tabular}{|c|c|c|c|c|}
\hline Parameter & №1 & №5 & №8 & Unit \\
\hline \multicolumn{5}{|c|}{$3 D$ parameters } \\
\hline $\mathrm{Sa}$ & 0.04 & 0.155 & 3,25 & $\mu \mathrm{m}$ \\
\hline Spk & 0.0135 & 0.129 & 2.91 & $\mu \mathrm{m}$ \\
\hline Sk & 0.0718 & 0.326 & 5.71 & $\mu \mathrm{m}$ \\
\hline Svk & 0.0154 & 0.137 & 1.9 & $\mu \mathrm{m}$ \\
\hline Ssk & 0.252 & -0.38 & 0.322 & - \\
\hline Str & 0.1211 & 0.0909 & 0.0983 & - \\
\hline Vmp & 0.0000211 & 0.000109 & 0.00165 & $\mathrm{~mm} 3 / \mathrm{mm} 2$ \\
\hline $\mathrm{Vm}$ & 0.000132 & 0.000789 & 0.00917 & $\mathrm{~mm} 3 / \mathrm{mm} 2$ \\
\hline $\mathrm{Vv}$ & 0.000116 & 0.000553 & 0.00966 & $\mathrm{~mm} 3 / \mathrm{mm} 2$ \\
\hline Vve & 0.0000662 & 0.00021 & 0.00541 & $\mathrm{~mm} 3 / \mathrm{mm} 2$ \\
\hline VvV & 0.0000325 & 0.0000267 & 0.000316 & $\mathrm{~mm} 3 / \mathrm{mm} 2$ \\
\hline $\mathrm{Sdr}$ & 0.000196 & 0.00708 & 1.55 & $\%$ \\
\hline $\mathrm{Sz}$ & 0.2 & 1.11 & 16.5 & $\mu \mathrm{m}$ \\
\hline Sku & 2.06 & 3.25 & 2.41 & - \\
\hline Smr1 & 5.83 & 11.7 & 11.4 & $\%$ \\
\hline Smr2 & 95.3 & 88.7 & 85.7 & $\%$ \\
\hline \multicolumn{5}{|c|}{$2 D$ parameters } \\
\hline RSml & 0.055 & 0.062 & 0.064 & $\mathrm{~mm}$ \\
\hline $\mathrm{RSm} 2$ & 0.041 & 0.057 & 0.071 & $\mathrm{~mm}$ \\
\hline
\end{tabular}

\section{ANALYSES OF ROUGHNESS PARAMETERS OF CYLINDRICALLY GRINDED SURFACES}

Roughness parameters of cylindrically grinded surfaces were divided into several groups, depending on the service properties of surfaces.

For the determination of surfaces' wear resistance it is useful to use parameters $S a$, Str, Ssk, Smrl and $S m r 2$. According to reference [6], the surface wear is characterized by mean arithmetic height of the surface, the roughness steps in the direction of $\mathrm{X}$ and $\mathrm{Y}$ axes, anisotropy coefficient and relation of nominal and actual area of the surface. And besides, the bigger are the surface irregularities $(\mathrm{Sa})$ the weaker is their resistance to deformation. In its turn the bigger are roughness steps values the greater area the protrusions will have, so under load they will be less exposed to shocks and deformations.

Table 2 shows that that sample No.1 has the least roughness and roughness step, which is connected with the reduced longitudinal feed rate of component. 
However asymmetry of roughness should also be taken into consideration, because it determines the distribution of load along peaks. Thus using parameter Ssk it is possible to determine the general shape of roughness peaks.

The surfaces No.1 and No.8 have positive values of parameter $S s k$, i.e. roughness micro-peaks have sharp form and are stress concentrators. At negative values of parameter Ssk peaks form flat micro-tops, so the load distribution area becomes larger.
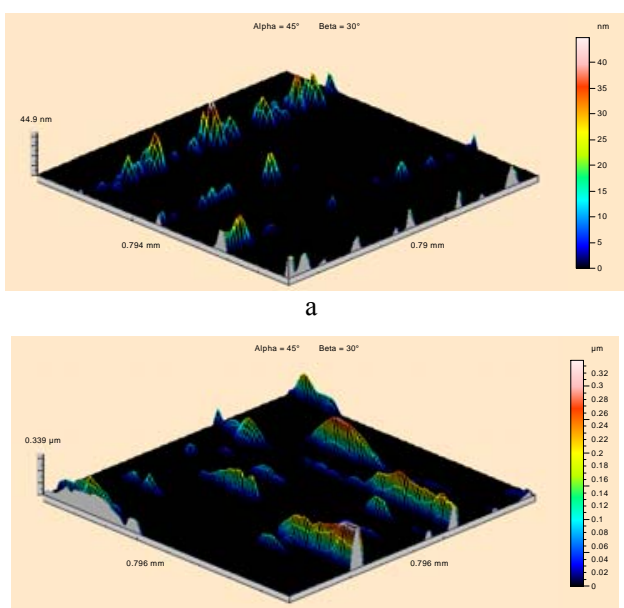

b

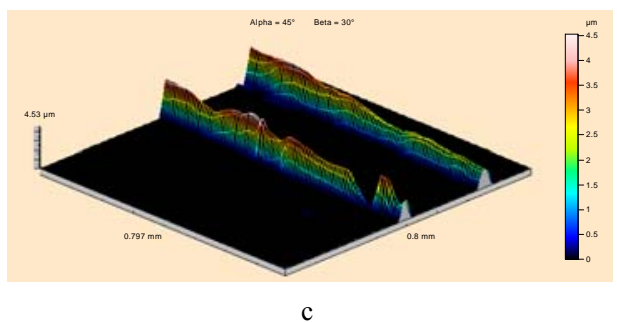

Fig.6. Volume of separated material of surfaces No.1 (a), No.5 (b), No.8 (c) during the running-in operation

Fig.6 shows a part of roughness, which will be rapidly separated from the surface during running-in initial stage of component exploitation. Values of parameter $V m p$ show the volume of the separated material, which is the biggest for the surface No.8. Possible meaning of wear can be determined also by the help of parameter Spk, which shows the height of the most pronounced micro-peaks.

Additionally it is needed to determine the contact area of surface. Generally, it is simpler to examine contact of rough surface with perfectly smooth (completely hard). In the initial stage of exploitation of component the surface contact takes place at definite points. But in the course of wear of the surface the contact area is increasing and after the runin stage the surface roughness can have the following look (Fig.7).

The area of bearing surface can be expressed in per cents using parameter Smrl, which for the surface
No. 1 is twice smaller than for No.5 and No.8. Thus it can be concluded that bigger values of roughness $S a$ testify more intensive wear, which practically is not affected by height distribution of roughness peaks.

Further in this work it was analyzed the ability of surface to retain lubricants, which mainly depends on hollows depth.

Understanding of the ability of surface to retain oils is given by parameters $V v, V v c$ and $V v v$, which help to determine the void volume of roughness prior to exploitation, after the running-in stage and after the period of normal wear.

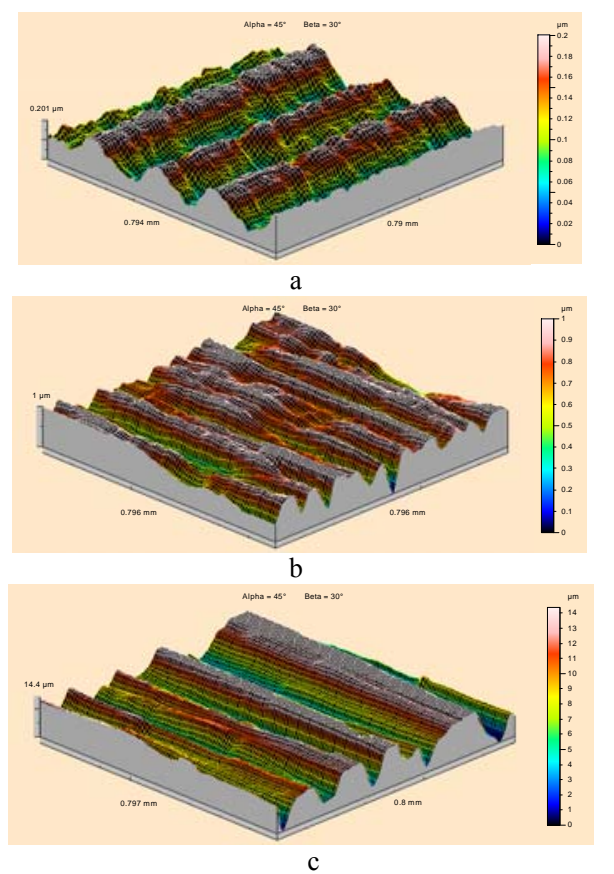

Fig.7. Roughness of surfaces No.1 (a), No.5 (b), No.8 (c) after running-in stage

The growth of height of micro-peeks as compared with optimum values increases wear, which is more intensive even at relatively low service loads, because on the tops of micro-peaks it is appeared a high specific pressure, which causes rupture of oil-film wedge and cutting off of micro-peaks. As a result the friction character changes, clearance becomes bigger, what brings to lubricants free extrusion and dynamic load formation. Due to the fact that the oil-film wedge task is to accommodate the substantial load, the oil shall be all the time evenly distributed between the surface irregularities.

The size of hollows of all three samples after run-in operation is reduced about twice. But the void volume of samples with higher roughness is considerably bigger. Yet this tendency was not observed while normal wear stage, during which values of hollows of surfaces No.1 and No.5 became practically similar. So it can be concluded that surfaces with explicit roughness possess an ability to retain lubrication. 


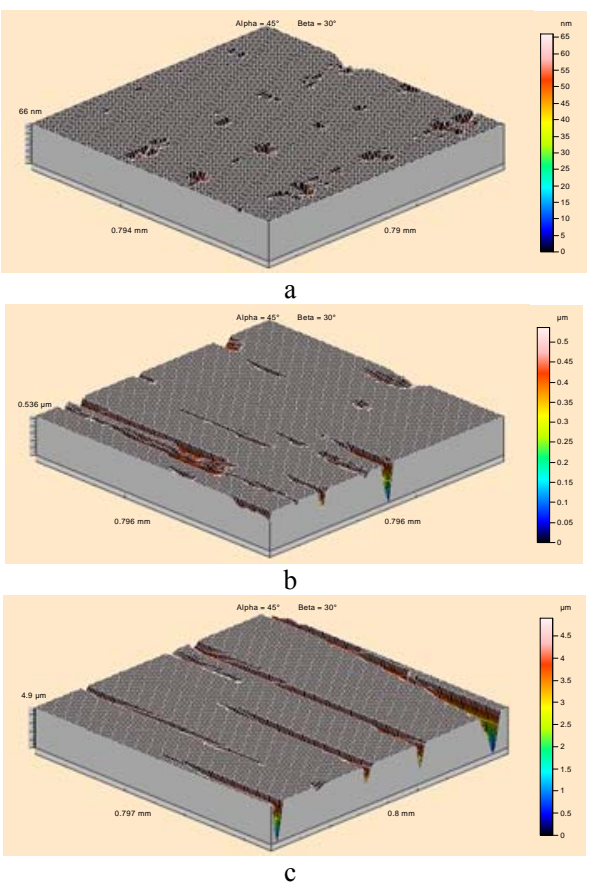

Fig.8. Roughness of surfaces No. 1(a), No.5 (b), No.8 (c) after intensive wear

Regarding to adhesion of coating it should be noted that the advantage of rough surface is the increase of area for the formation of adhesion connections, because the large surface area of substrate provides a greater number of links. For this purpose, it is important to know the value of the parameter $S a$, which gives a notion of the arithmetic average height of roughness and surface smoothness. To understand how great is contact area of deposited coating, it is important to know the value of parameter $S d r$, which is showing relations between nominal surface (without roughness) and actual surface. The bigger the area of actual surface compared to nominal, the more connections will be formed between the substrate and deposited coating.

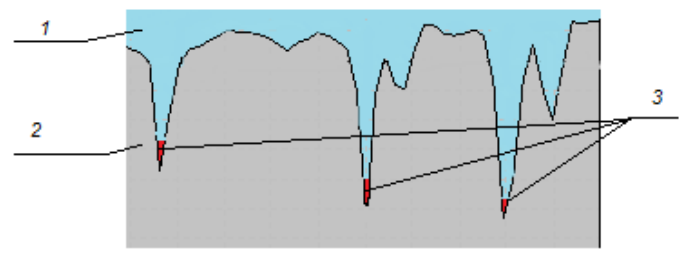

Fig.9. Formation of air pocket. 1 - coating, 2 - substrate, 3 - air pocket [7]

Because area of substrate surface depends on the character of roughness, it is necessary that surface has deep valleys. Yet there is a risk of air capturing [7]. Air capturing can considerably reduce the efficient volume for sticking as a result connections will be weakened. (Fig.9.). Therefore to allow adhesive to flow into these valleys their form should be rounded as possible. The distribution of surface roughness height is characterized by parameter $S k u$, which values, less than 3 , indicate that surface has no sharp peaks.

Parameter $S d r$ of surfaces No.1 and No.5 has very small values, only at a considerable increase of roughness the necessary surface area can be ensured for retaining coatings. Values of parameter $S v k$ indicates that surface No.3 has rather deep hollows but this does not ensure good adhesion, since hollows have sharp form, what is indicated by the values of parameter $S k u$. All three samples have the same problem.

\section{CONCLUSION}

In the course of research mathematical model of cylindrically ground surfaces was determined, including diminishing correlation function and distribution density of ordinates of the surface, which corresponds to normal distribution law.

Roughness of the surface by the increase of the longitudinal feed rate becomes more explicit and the values of mean arithmetical height of roughness are increasing respectively. The given relation affects negatively the wear resistance of the surface however the values of volume of voids to retain lubricant can be sufficient.

It should also be noted that roughness ensures a larger area for its adhesion with a coating, but there is risk of emerging air pocket because of the sharp shape of valleys. With the growth of roughness the average step of roughness is also increasing, what positively affects the distribution of load while surfaces contacting.

\section{ACKNOWLEDGMENTS}

This work has been supported by the European Social Fund within the project "Support for the implementation of doctoral studies at Riga Technical University".

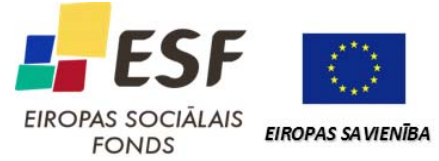

\section{REFERENCES}

[1] ISO 25178-2:2012, Geometrical product specifications (GPS) - Surface texture: Areal - Part 2: Terms, definitions and surface texture parameters.

[2] Taylor Hobson Ltd. "Form Talysurf Intra", Taylor Hobson Precision, 2013. - 12 p.

[3] K.J.Stout, P.J.Sullivan, W.P.Dong, E.Mainsah, N.Luo, T.Mathia, H.Zahouani, Development of Methods for the Characterisation of Roughness in Three Dimensions, ECSCEEEC-AEC, Brussels - Luxemburg, 1993. - 359 p.

[4] A.N.Tabenkin., S.B.Tarasov, S.N.Stepanov, Roughness, waveness, profile, $\mathrm{SPb}$ : Politeh. un., 2007-136 p.

[5] J.Rudzitis, Contact mechanics of surfaces, part I. RTU, Riga, 2007. - 193p. 
Natalija Bulaha / Environment. Technology. Resources, (2015), Volume I, 16-21

[6] J.Rudzitis, Contact mechanics of surfaces, part II. RTU, Riga, 2007. $-213 p$
[7] N.Filipova, Surface texture parameters application, studying plane grinded surfaces. Master's work, RTU, 2014. - 79 p. 